\title{
Home Energy Management as a Service over Networking Platforms
}

\author{
Korosh Vatanparvar, Quan Chau, Mohammad Abdullah Al Faruque \\ Department of Electrical Engineering and Computer Science \\ University of California, Irvine \\ Irvine, California, USA \\ Email: \{kvatanpa, alfaruqu\} @ uci.edu
}

\begin{abstract}
About $40 \%$ of the U.S. primary energy is consumed in the buildings. Therefore, to manage the energy consumption and its usages pattern, for instance, the U.S. state of California mandated Zero Net Energy buildings by 2020 for the residential sector (22\% of the U.S. primary energy). Moreover, towards this goal, U.S. Department of Energy, Building Technologies Office is trying to develop techniques to improve the efficiency of buildings. Home Energy Management (HEM) may be used to improve the energy consumption in the residential buildings. However, the cost, scalability, and flexibility of the software and hardware architectures available, have increased the time-tomarket and have made HEMs hard to penetrate the consumer market. To solve these limitations, in this paper, we have presented a novel HEM platform, which utilizes a low power IEEE 802.15.4 standard to monitor and control all the appliances in a house. Moreover, it utilizes Service-Oriented Architecture and Devices Profile for Web Services to implement the monitoring and controlling algorithms and therefore may significantly improve the scalability and flexibility of the platform. Also, some other features like: plug-n-play capability, remote access feature, and open source architecture are implemented in this platform. Finally, a table-top prototype of the HEM platform is demonstrated in the paper.
\end{abstract}

Index Terms-Home energy automation, Energy management, Software as a service, Control as a Service.

\section{INTRODUCTION AND RELATED WORK}

In the year 2013, about $40.7 \%$ of the U.S. primary energy consumption is due to buildings share [1]. Reducing the energy consumption of the buildings may help to decrease this number significantly. For instance, in the U.S. state of California, the California Energy Commission, planned to reach Zero Net Energy (ZNE) buildings by 2020 [2]. Also, the U.S. Department of Energy (DOE) Building Technologies Office (BTO) seeks to develop technologies and techniques to improve the efficiency of new and existing residential and commercial buildings to reduce the national energy consumption [3]. Therefore, the need for efficient buildings is growing rapidly. Moreover, the advancement in technology, the possibility of integrating low power and high performance electronic components to build advanced embedded systems, and reduction in the cost and size of devices like: sensors, actuators, network adapters, switches, etc., have provided us with the opportunity to build smart houses [4]. These houses may be remotely monitored and controlled by the Utilities and the consumers. Also, to reduce the energy consumption and the energy costs, periodic energy cost feedback and remote monitoring for control may be provided [5]. This ability may be integrated as Demand Response (DR) program [6]. As a result, a platform needs to be implemented in the houses to monitor various appliances (e.g. water heater, HVAC, dryer, etc.) and control them to reduce energy consumption. This integration of different hardware, software, and communication architectures towards building an energy efficient house is called, Home Energy Management (HEM) platform.

The hardware, software, and communication architecture required for HEM platform needs to follow different policies and rules to make it economical for mass production. One of the most important properties to be considered is the probability of penetrating into the consumer market and affordability of the platform for an ordinary consumer. Major requirements for the architecture which influence this penetration and affordability are: 1) interoperability; 2) scalability; 3) ease of deployment; 4) open architecture; 5) plug-n-play capability; and 6) local and remote monitoring [7] [8]. Moreover, meeting these requirements in a single package should also be cost-effective. While different hardware, software, communication architectures have been proposed and compared by their power consumption, performance, etc. [6] [9] [10] [11] [12] [13], the cost of implementing the infrastructure like: computing devices, software stack, communication devices, etc. are still high enough that hinder the process of implementing the smart home for ordinary users. Moreover, the hardware and software architectures may not be able to handle the growing number of sensors and actuators with their heterogeneity. Therefore, by implementing monitoring and controlling sections of the HEM platform using web services (service-oriented architecture), we may reach the agility, flexibility, scalability, and other features required for a feasible and affordable HEM platform [8] [9] [10].

Rest of the paper is organized as follows: Section II explains the contributions which have been made by presenting the HEM platform. Section III states different purposes and applications for the HEM platform. Section IV explains in further detail the architecture of the platform, also it discusses about some of the advantageous of the platform. Section V demonstrates a prototype of a HEM platform which we have built and finally Section VI concludes the paper.

\section{OUR NOVEL CONTRIBUTIONS}

This paper presents a platform which contributes to the main requirements for an HEM platform in the ways listed below:

1) The platform is composed of heterogeneous subsystems which communicate and synchronize with each other to 
establish a coherent monitoring and controlling platform (interoperability).

2) Deploying the open source software architecture and hardware infrastructure (open architecture) built on a networking platform provides us with the ability to scale the platform (scalability).

3) Leveraging low power IEEE 802.15.4, ZigBee [15] standard for communication between devices, subsystems, and low power, open source sensor nodes.

4) The software for the subsystems or devices is implemented as services using Devices Profile for Web Services (DPWS) [8] [10] [14] which also used for the discovery purpose to provide the plug-n-play feature.

5) Building a prototype of the HEM platform, demonstrating the local and remote monitoring, controlling, and features using web-based user interface, and managing different devices in the house.

\section{APPLICATIONS FOR THE PLATFORM}

The platform may be used for different types of buildings to manage their energy. The energy management may have different purposes like: 1) monitoring and metering the energy consumption of each device in the house to estimate the monthly bill; and 2) implement algorithms for managing the smart devices efficiently, e.g. intelligent lighting, EV charger [16], HVAC management, smart refrigerator, etc.

\section{The PlatForm ARChitecture}

The platform is composed of system of systems. The architectures of the systems may be categorized into hardware, software, and communication. Each category will be explained in details:

\section{1) The Hardware Architecture}

A house includes various appliances. These appliances may have the feature to establish a connection with the user and provide them with the monitoring and controlling capabilities. Therefore, the appliances with these capabilities are smart enough to be monitored and controlled locally or remotely by a HEM platform ${ }^{1}$.

The main part of the HEM platform is the monitoring and controlling part called "HEM Control Panel". The main job of it is to discover and monitor different devices in the network and handle different time-flexible load requests and trigger and command the devices accordingly based on the algorithms implemented. The HEM control panel may be implemented on an open source user-configurable router. Also, the ports of communication installed in the routers, enable the developer to connect other subsystem or appliances with any possible protocol or standard. In other words, the smart devices may use a proprietary protocol or even a standard protocol not supported by the router, e.g. ZigBee. The conversion and forwarding process of the messages between different protocols may be done by a simple translator device (relays the messages in different protocol) attached to the router, e.g. a Raspberry Pi may be used to translate from Ethernet to

\footnotetext{
${ }^{1}$ It is assumed that the appliances are available and connected to the network in the house. However, the type of the appliances and their existence are orthogonal to the platform.
}

ZigBee standard. This "Gateway" device is the point of connection between the HEM control panel and the smart devices or subsystem of devices. Also, to extend the network, some devices may act as a "switch" to make a hierarchical network and directly exchange the messages between different layers of hierarchy. Moreover, the smart devices have their own monitoring and controlling part. The "Control Panel" provides the user with the capability of configuring the device in lower level and much more detail than the HEM control panel may be capable of.

To manage the energy consumption of the house, the HEM platform might need to retrieve information about the environment of the house. The physical world condition may be monitored by multiple sensors (e.g. temperature, humidity, light, gas, etc.) in different places of the house. Moreover, different types of sensors may be implemented on a single device (e.g. TelosB mote module).

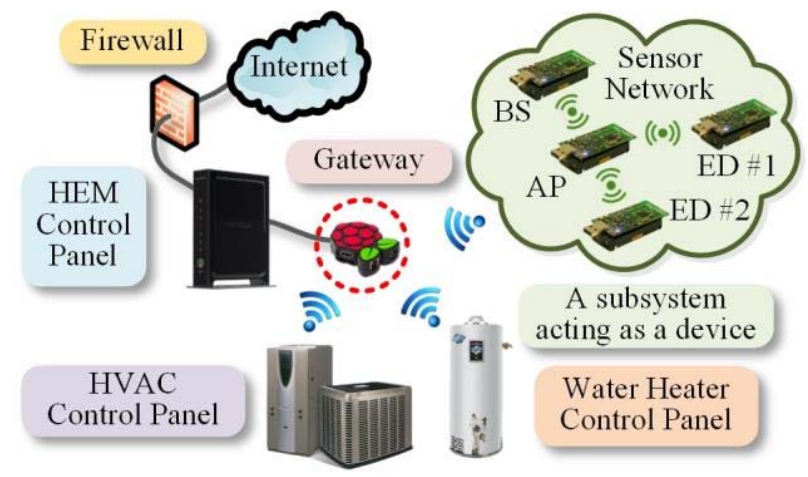

Figure 1. The hardware architecture of the HEM platform. As you may see, the "Gateway" may be eliminated, if the appliance or subsystem is capable of communicating with the platform directly.

2) The Software Architecture

The HEM control panel needs to process all the information retrieved from other devices or sensors in the network, and then using the control algorithms implemented, decides how to manage the devices. The open source and userconfigurable routers run a distribution of Linux on a MIPS processor. This feature helps the developer of the control algorithms to easily code, compile, and run them on the router.

The sensor devices, e.g. TelosB mote modules are compatible with TinyOS [17] which is an open source operating system designed for low power wireless sensors. Therefore, the algorithms designed for monitoring, commanding the sensors and receiving data from them may be coded easily and dynamically on the sensors. This flexibility may help the developers to program the sensors based on their requirements. In other words, different routing and discovery algorithms or different kinds of sensors (e.g. temperature, humidity, etc.) may be implemented in different situations for different buildings.

The control panels for each subsystem manage their devices in the network through a defined protocol. However, all the subsystems and devices connected in the main network need to follow a unique protocol defined by the HEM control panel (e.g. ZigBee). Moreover, the communications between devices are built around DPWS from the Web Services for 
Devices (WS4D). Also, it relies on SOAP-over-UDP, SOAP, WSDL, and XML Schema. This protocol stack may be used for sending secure messages from device to device in a heterogeneous platform. The developers may utilize the WS4D-gSOAP toolkit to implement the services needed for each device. Devices may host various DPWS-compliant services using:

1) WS-Addressing: provides an addressing mechanism for web services as well as messages in a transportneutral matter.

2) WS-Discovery: provides a way to make the services discoverable by leveraging a protocol based on IP multicast.

3) WS-MetadataExchange: defines different data types and the operations to receive a metadata.

4) WS-Transfer: is used to transfer the metadata and is very similar to HTTP.

5) WS-Eventing: describes a protocol which allows web services to subscribe to or accept subscriptions for event notification messages.

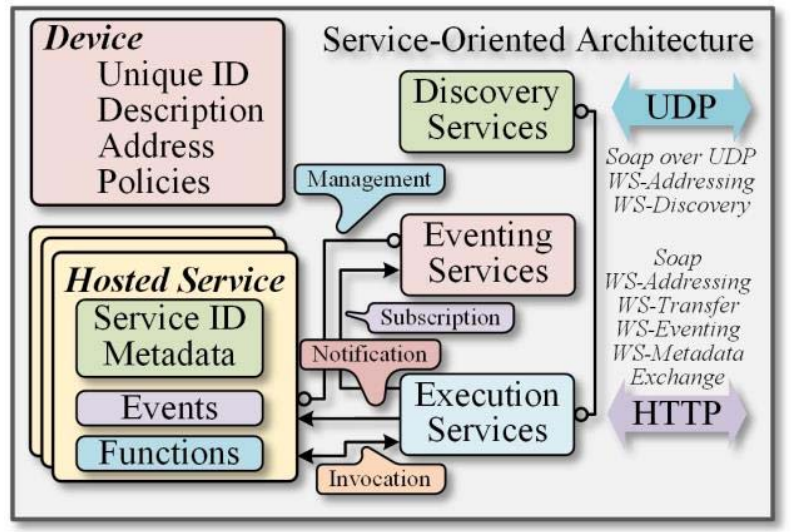

Figure 2. The demonstration of the software architecture which is implemented by Service-Oriented Architecture (SOA). Each device has its own registered hosted services.

Since the web services are platform-agnostic, using Service-Oriented Architecture (SOA), the platform maintains its flexibility of adding new devices, discovering devices and their respected services, synchronization between devices, and transferring structured data between them. Also, in HEM platform, the appliances, devices, or the controlling algorithms required by a user may be added and managed as a service (Control as a Service). Moreover, to establish synchronization capability and interoperability between devices in the network, e.g. HEM control panel, the device may host 'eventing' web services. In this way, the devices will be notified of any changes or events related to other devices if they have subscribed to those events.

\section{3) The Communication Architecture}

The routers in the house network may have different network adapters and interfaces, e.g. Wireless, Ethernet, Bluetooth, Universal Serial Bus (USB), etc., based on their specifications. Since a unique protocol (e.g. ZigBee) should be used for the main network, these interfaces need to be converted using gateway devices for the desired protocol if needed.
The sensor devices, e.g. TelosB mote modules, may use the standards-based, low power wireless technology - ZigBee - to communicate with each other. Moreover, the standard used for the communication between different nodes may be Smart Energy Profile (SEP 2.0), which is a standard for IPbased control for home energy management and it is supported by these devices, but this profile is not ready yet (standard, protocol stack and hardware). Furthermore, the flexibility of the ZigBee networks can handle about 65535 devices in a network. All the devices are tagged using a unique Identification (ID) number. These IDs are hard coded into the sensor devices while programming and maybe used for routing and transferring data in the same network.

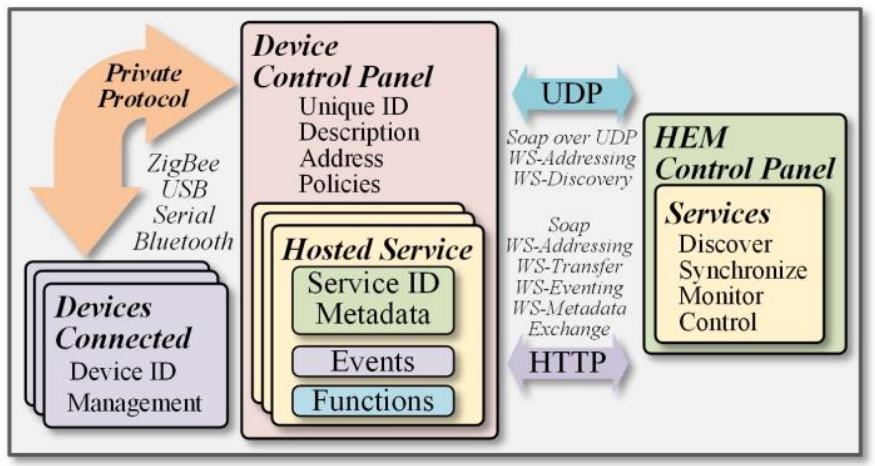

Figure 3. The communication architecture of the platforn and the protocols used for each section of the platform.

To further extend the range that sensor network supports, we may utilize a two-level hierarchical network. In other words, some of the sensor devices are programmed to only sense which are called End Devices (ED) and may be placed in different corners in a room. Then, a sensor node may be programmed to act as an Access Point (AP) and connects to all the end devices in that room. Also, in a higher level, another sensor device may act as a Base Station (BS) which connects to multiple access points in all the rooms. The end devices transfer the data through their access points and then to the base station. Moreover, the number of access points connected to each base station or the number of end devices connected to the access points are the variables which may be adjusted based on the structure of the building and the requirements.

The base station which transfers the data gathered from all the end devices in the sensor network, may directly communicate with the HEM control panel, if it is compatible. On the other hand, it may communicate with the HEM control panel indirectly through a compatible gateway, e.g. the sensor nodes may communicate with a Raspberry Pi using serial connection over ZigBee standard, and then the Raspberry Pi connects to the HEM Control Panel through Ethernet. However, if the router has the capability of communicating via ZigBee directly, or the router had the compatible driver to control the sensor module through USB, the Raspberry Pi would be eliminated.

Furthermore, the user may interface with the platform using the web pages designed for the control panel of each device. The HEM control panel interface is setup on the main router. However, the interfaces for other devices may be 
provided by the vendors of the devices. Moreover, Asynchronous JavaScript and XML (AJAX) technique may be utilized to retrieve the information from the devices and view it on the web page and trigger different functions implemented on the control panel to process input data. Furthermore, connecting the HEM control panel to the Internet enables the user to monitor and control the house locally or remotely through internet.

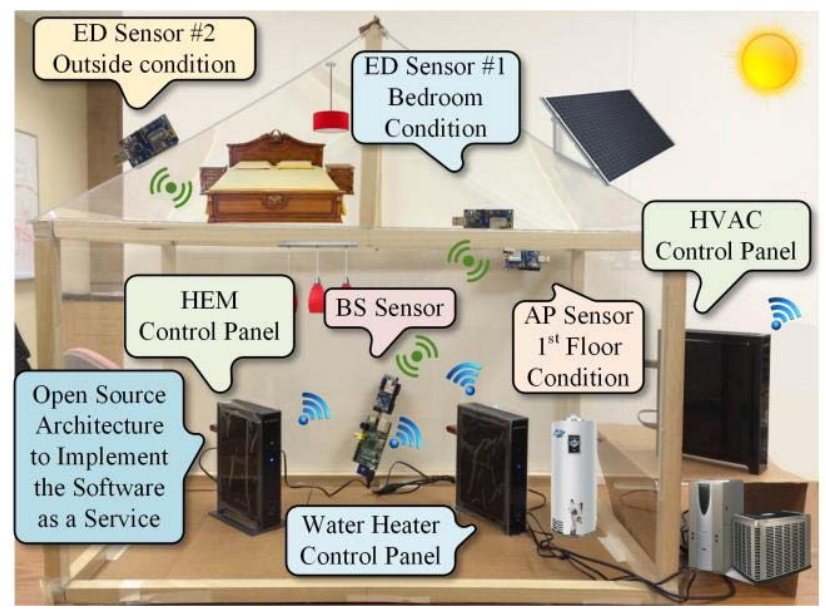

Figure 4. The house prototype demonstrating the Home Energy Management platform using the proposed platform. The appliances are modeled in the open source routers.

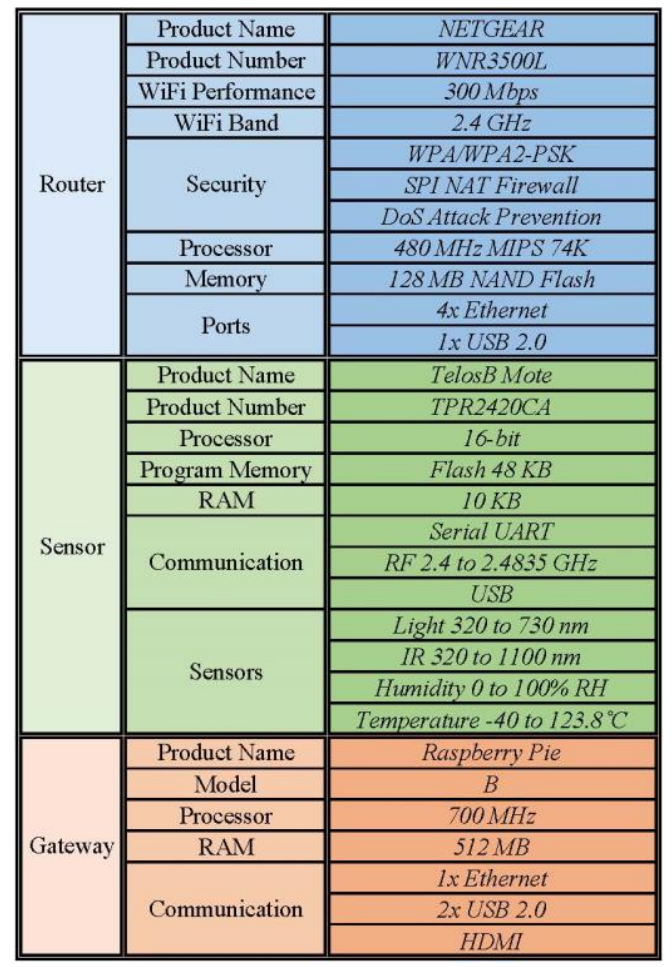

Figure 5. The hardware specification of the prototype.

\section{4) The Integration of Architectures}

As we have explained in the last three subsections, the flexibility and low cost infrastructure helps us to add any device, sensor, actuator, and their respected services. The services for the devices have to be defined using WS4D to be able to communicate with the routers and each other. On the other hand, if a subsystem designed for an application-specific purpose, needs to be added to the platform, regardless of its own protocol and architecture, it needs to be compatible with the protocol stack used in the HEM platform, or we can make it compatible by using gateway devices.

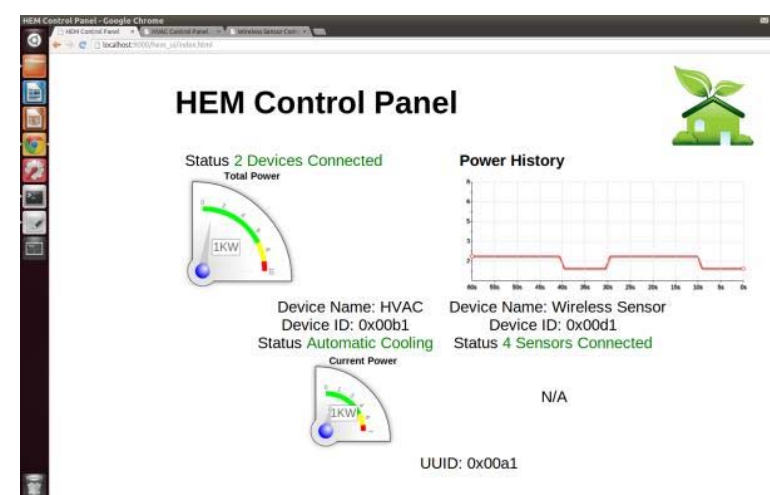

Figure 6. Through the HEM control panel, the user may view the status of all the appliances which have been discovered.

\section{PROTOTYPE AND DEMONSTRATION}

To test this platform for the purpose of Home Energy Management (HEM), the following architecture has been implemented for one house as shown in Fig. 4. In the house, multiple smart appliances such as: HVAC, water heater, refrigerator, washer, and dryer are implemented. Each appliance is monitored and controlled by the HEM control panel. Also, they have their own control panels to monitor the status of the devices and set their configurations. In this prototype, all the appliances are software model. However, in the real-world implementation, the control panels of the appliances will be provided by their vendors.

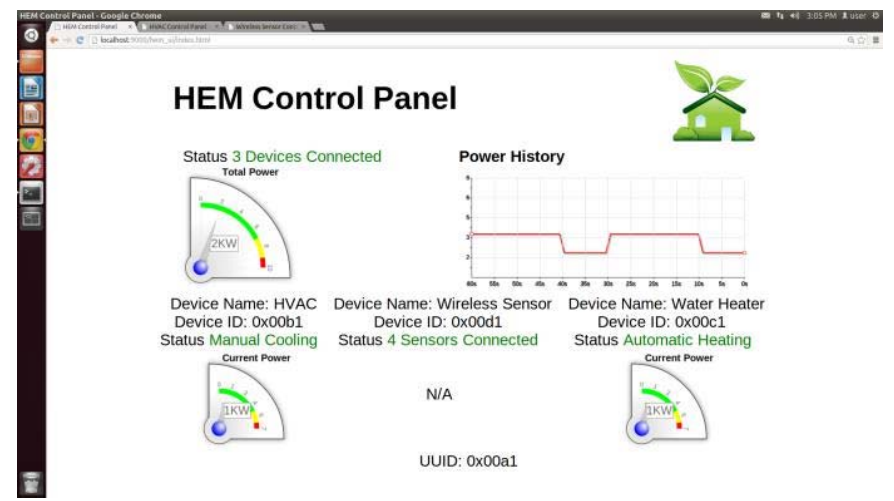

Figure 7. The HEM control panel after pluging in the water heater and changing the configuration of the HVAC to manual.

Moreover, a room is being monitored by a network of four sensor devices (TelosB mote modules). The sensor network is defined as a subsystem inside the platform (system of systems). They sample the temperature and humidity of the room. Also, different types of commands to enable/disable the sensors or set the configurations, e.g. sampling rate for the sensors, may be sent by the control panel connected to the base station to each device by specifying the ID number of that device. 


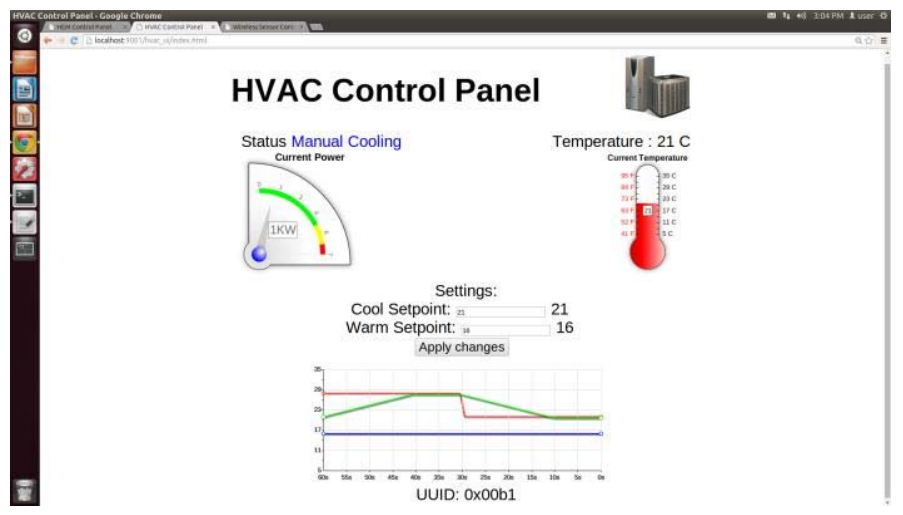

Figure 8. Through the HVAC control panel, the user may easily monitor and control the HVAC. This may be provided by the vendor.

The user may interact with the control panels using web pages running. It is shown in Fig. 6, that the HEM control panel monitors all the devices discovered in the house. When a new device is added or a status is changed it may be shown in the list (Fig. 7). Moreover, as shown in Fig. 8, the user may use the HVAC control panel to view the power consumption of the HVAC and the room temperature, and decide how to configure temperature set points manually. In Fig. 9, the configurations for the sensor network may be set by the user. The user may specify the sampling rate of the sensors and enable/disable a specific sensor.

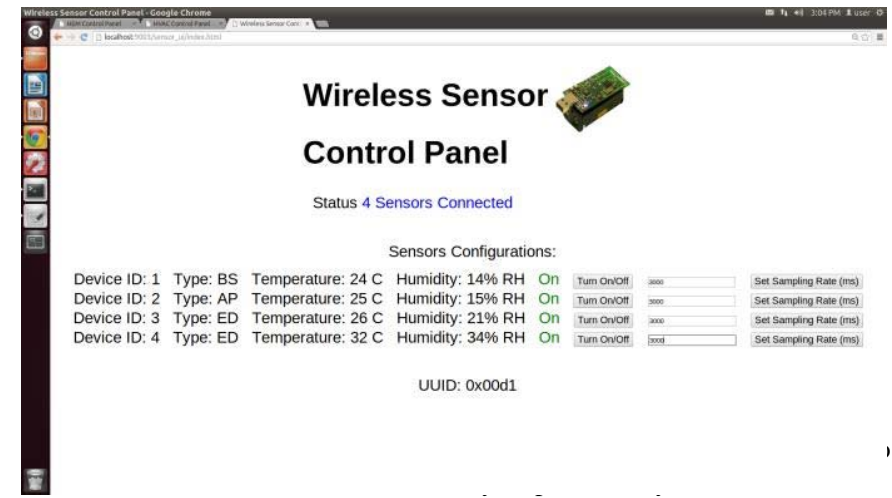

\section{CONCLUSION}

In the proposed HEM platform, the hardware architecture has been implemented on a flexible, scalable, low cost, and low power infrastructure. Moreover, adding or removing devices in the house is performed by connecting them to the main network of the platform, e.g. ZigBee. Also, the control algorithms required by the user may be implemented as services by registering them in the open source routers, e.g. the HEM control panel. Furthermore, the SOA provides the flexibility and the agility a Home Energy Management platform demands. On the other hand, since the platform is setup using off-the-shelf open source routers which may already exist in some ordinary houses or they are cheap to buy, the total cost decreases significantly. Moreover, the webbased user interface makes it more convenient for a user to monitor and control the house and the appliances locally or remotely.

\section{ACKNOWLEDGEMENT}

We would like to appreciate MAXFOR Technology Inc. [18] for providing us with the wireless sensor modules. Also, we thank Tahmid Khan, a K-12 student from University High, Irvine, CA for his help to build the house prototype.

\section{REFERENCES}

[1] Department of Energy (DOE), "Buildings Energy Data Book". [Online]. Available: http://buildingsdatabook.eren.doe.gov/TableView .aspx?table=1.1.3

[2] California Energy Commission, "Building Energy Efficiency Program". [Online]. Available: http://www.energy.ca.gov/title24/

[3] Department of Energy (DOE), "US Department of Energy Strategic Plan". [Online]. Available: http://energy.gov/sites/prod/files/ 2011_DOE_Strategic Plan_pdf

[4] H. Farhangi. "The path of the smart grid." Power and Energy Magazine, IEEE 8, no. 1 (2010): 18-28.

[5] D. Niyato, L. Xiao, and P. Wang. "Machine-to-Machine Communications for Home Energy Management System in Smart Grid." IEEE Communications Magazine, 49, no. 4 (2011): 53-59.

[6] P. Palensky and D. Dietrich. "Demand Side Management: Demand Response, Intelligent Energy Systems, and Smart Loads." IEEE Transactions on Industrial Informatics, 7, no. 3 (2011): 381-388.

[7] U.S. Department of Energy Energy Efficiency and Renewable Energy Golden Service Center. Financial Assistant Funding Oppurtunity Announcement. 28 March 2013. "DE-FOA-0000822: "Turn Key" Open Source Software Solutions for Energy Management of Small to Medium Sized Buildings".

[8] F. Jammes, and H. Smit. "Service-Oriented Paradigms in Industrial Automation." IEEE Transactions on Industrial Informatics, 1, no. 1 (2005): 62-70.

[9] Bian, D., M. Kuzlu, M. Pipattanasomporn, and S. Rahman. "Assessment of Communication Technologies for a Home Energy Management System." In Innovative Smart Grid Technologies Conference (ISGT), IEEE PES, pp. 1-5. IEEE, 2014.

[10] M. M. Rahman, M. Kuzlu, M. Pipattanasomporn, and S. Rahman. "Architecture of Web Services Interface for A Home Energy Management System." In Innovative Smart Grid Technologies Conference (ISGT), IEEE PES, pp. 1-5. IEEE, 2014.

[11] D. Han and J. Lim. "Design and Implementation of Smart Home Energy Management Systems based on ZigBee." IEEE Transactions on Consumer Electronics, 56, no. 3 (2010): 1417-1425.

[12] Y. Son, T. Pulkkinen, K. Deok Moon, and C. Kim. "Home Energy Management System based on Power Line Communication." IEEE Transactions on Consumer Electronics, 56, no. 3 (2010): 1380-1386.

[13] S. Katipamula, R. M. Underhill, J. K. Goddard, D. Taasevigen, M. A. Piette, J. Granderson, R. E. Brown, S. M. Lanzisera, and T. Kuruganti. "Small-and Medium-Sized Commercial Building Monitoring and Controls Needs: A Scoping Study." Pacific Northwest National Laboratory (PNNL), Richland, WA (US), Tech. Rep (2012).

[14] E. Zeeb, A. Bobek, H. Bohn, and F. Golatowski. "Service-Oriented Architectures for Embedded Systems Using Devices Profile for Web Services." In Advanced Information Networking and Applications Workshops, 2007, AINAW'07. 21st International Conference on, vol. 1, pp. 956-963. IEEE, 2007.

[15] P. Baronti, P. Pillai, V. WC Chook, S. Chessa, A. Gotta, and Y. Fun Hu. "Wireless sensor networks: A survey on the state of the art and the 802.15.4 and ZigBee standards." Computer communications 30, no. 7 (2007): 1655-1695.

[16] M. A. Al Faruque, L. Dalloro, S. Zhou, H. Ludwig, and G. Lo. "Managing residential-level EV charging using network-as-automation platform (NAP) technology." In Electric Vehicle Conference (IEVC), 2012 IEEE International, pp. 1-6. IEEE, 2012.

[17] P. Levis, S. Madden, J. Polastre, R. Szewczyk, K. Whitehouse, A. Woo, D. Gay et al. "TinyOS: An Operating System for Sensor Networks." In Ambient intelligence, pp. 115-148. Springer Berlin Heidelberg, 2005.

[18] MAXFOR Technology Inc.. "Products / WSN modules". [Online]. Available: http://www.maxfor.co.kr 